\title{
Time-Resolved Indirect Nanoplasmonic Sensing Spectroscopy of Dye Molecule Interactions with Dense and Mesoporous $\mathrm{TiO}_{2}$ Films
}

\author{
Viktoria Gusak, ${ }^{\dagger}$ Leo-Philipp Heiniger, ${ }^{\dagger}$ Michael Graetzel, ${ }^{\ddagger}$ Christoph Langhammer, ${ }^{*},, \S$ \\ and Bengt Kasemo* ${ }^{\dagger}$ \\ ${ }^{\dagger}$ Department of Applied Physics, Chalmers University of Technology, SE-412 96 Göteborg, Sweden \\ ${ }^{\ddagger}$ Laboratory of Photonics and Interfaces, Institute of Chemical Sciences and Engineering, Ecole Polytechnique Fédérale de Lausanne, \\ Station 6, CH-1015 Lausanne, Switzerland \\ ${ }^{\S}$ Insplorion AB, Stena Center 1C, SE-412 96 Göteborg, Sweden
}

\section{Supporting Information}

\begin{abstract}
Indirect nanoplasmonic sensing (INPS) is an experimental platform exploiting localized surface plasmon resonance (LSPR) detection of processes in nanomaterials, molecular assemblies, and films at the nanoscale. Here we have for the first time applied INPS to study dye molecule adsorption/ impregnation of two types of $\mathrm{TiO}_{2}$ materials: thick $(10 \mu \mathrm{m})$ mesoporous films of the kind used as photoanode in dye-sensitized solar cells (DSCs), with particle/pore size in the range of $20 \mathrm{~nm}$, and thin $(12-70 \mathrm{~nm})$, dense, and flat films. For the thick-film experiments plasmonic $\mathrm{Au}$ nanoparticles were placed at the hidden, internal interface between the sensor surface and the mesoporous $\mathrm{TiO}_{2}$. This approach provides a unique opportunity to selectively follow dye adsorption locally in the hidden interface region inside the material and inspires a generic and new type of nanoplasmonic hidden interface spectroscopy. The specific DSC measurement revealed a time constant of thousands of seconds before the dye impregnation front (the diffusion front) reaches the hidden interface. In contrast, dye adsorption on the dense, thin $\mathrm{TiO}_{2}$ films exhibited much faster, Langmuirlike monolayer formation kinetics with saturation on a time scale of order $100 \mathrm{~s}$. This new type of INPS measurement provides a powerful tool to measure and optimize dye impregnation kinetics of DSCs and, from a more general point of view, offers a generic experimental platform to measure adsorption/desorption and diffusion phenomena in solid and mesoporous systems and at internal hidden interfaces.
\end{abstract}

KEYWORDS: Dye-sensitized solar cell, mesoporous titanium dioxide, localized surface plasmon resonance, indirect nanoplasmonic sensing, hidden interface

$\mathrm{T}$ he properties and organization of dye molecules on the surfaces of the mesoporous $\mathrm{TiO}_{2}$ anode of dye sensitized solar cells (DSCs) play a crucial role for the function of the DSC. Relevant properties are the density of adsorbed dye molecules, their mode of anchoring to the surface, and their electronic interaction with the $\mathrm{TiO}_{2}$ surface that determines interfacial electron-transfer rates. It is therefore very important to examine, understand, and control the surface self-assembly process of the dye molecules, including the kinetics at the $\mathrm{TiO}_{2}$ interface, as recently demonstrated by a $20 \%$ increase in cell performance after consecutive dye adsorption cycles. ${ }^{1}$ Details regarding to what extent optimized dye adsorption may boost performance are to date poorly understood and are the main motivation for developing the experimental methods presented here.

The mesoporous anode structure of a DSC is impregnated with dye molecules by exposing the anode to a dye solution. Mechanistically, the process is a combination of pore diffusion and adsorption/desorption events. The impregnation begins in the top layer of the porous structure, and then as time elapses, it reaches deeper and deeper, until eventually the whole sample is saturated with dye molecules. The interplay of the diffusion/ adsorption/desorption processes determines both the overall impregnation process and, for a given anode material, the resulting properties of the dye (mono)layer in the mesoporous structure. Specific quantities of practical interest are the impregnation time to reach saturation, the saturation coverage, how the latter can be optimized, e.g., by using different dye concentrations, the influence of sequential adsorption/rinsing/ adsorption steps or coadsorbates, and how stable the monolayer structure is toward desorption (i.e., the binding strength) or displacement by solvent molecules.

The amount of dye adsorbed is usually measured by means of optical absorption spectroscopy. Other real time techniques,

Received: January 30, 2012

Revised: April 7, 2012

Published: April 9, 2012 
such as electrochemistry, ${ }^{2}$ very recently quartz crystal microbalance with dissipation (QCM-D), ${ }^{3}$ and optical waveguide spectroscopy ${ }^{4}$ have also been used. All these experimental approaches have in common that they monitor the integral amount of dye molecules adsorbed by the porous photoanode and, hence, do not allow any conclusions about the local adsorption kinetics, e.g., at the inner hidden interface between the porous film and the collecting electrode or in the bulk of the material.

Here we present, for the first time, hidden interface indirect nanoplasmonic sensing (which we call HI-INPS) to study the dye-impregnation kinetics of DSCs. The underlying INPS methodology is described in detail below. As the first step, we used the standard INPS experimental platform to analyze dye adsorption on thin, nonporous $\mathrm{RF}$-sputtered $\mathrm{TiO}_{2}$ films (Figure 1a), which we in the following call dense or flat films. These
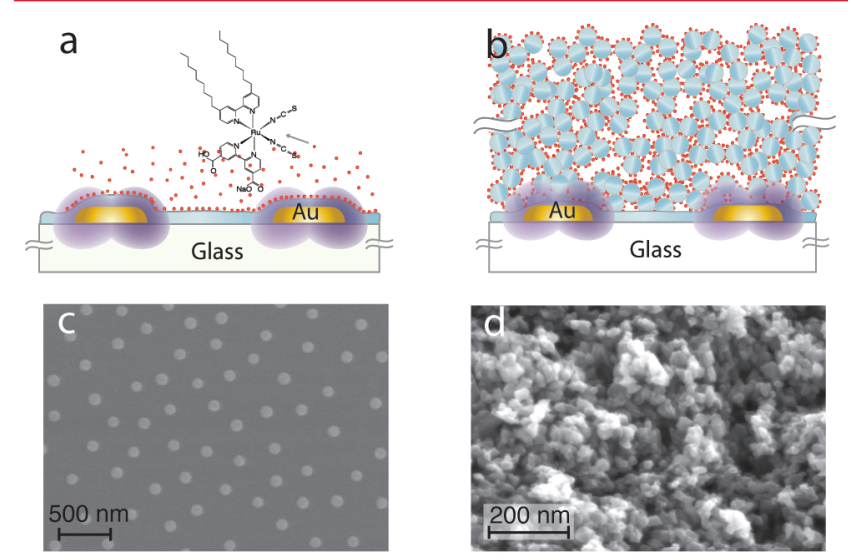

e

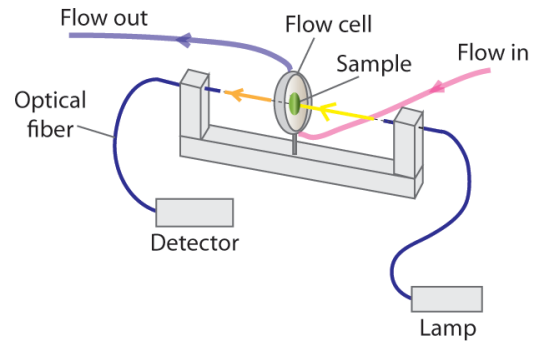

Figure 1. Sensor chip structure used for studies of dye adsorption on (a) dense (flat) and (b) mesoporous $\mathrm{TiO}_{2}$ films. (c) SEM images of $\mathrm{Au}$ nanoparticles covered with a $12 \mathrm{~nm}$ thin, dense $\mathrm{TiO}_{2}$ film. (d) A mesoporous $\mathrm{TiO}_{2}$ film (the Au nanoparticles are at the bottom of the latter film and are not visible in the SEM image). (e) Schematics of the optical absorption measurement setup used in this work.

experiments are ideal to determine monolayer adsorption kinetics and amount (density) of surface loading with dye molecules under different conditions. Langmuir-like first-order adsorption kinetics was found for the studied system. Furthermore, by repeating the dense film experiment with systematic variation of the thickness of the $\mathrm{TiO}_{2}$ film, the spatial extension of the enhanced local electromagnetic field that is key to LSPR sensing could be obtained, i.e., we obtained a measure of the sensing volume. The latter was found to extend ca. 70 nm from the Au nanoparticle sensor surface into the $\mathrm{TiO}_{2}$.

As a second step, we measured the dye molecule adsorption kinetics inside a three-dimensional (3D) $10 \mu \mathrm{m}$ thick mesoporous $\mathrm{TiO}_{2}$ photoanode by placing plasmonic $\mathrm{Au}$ nanodisk sensors at the interface between the mesoporous $\mathrm{TiO}_{2}$ and the glass support (Figure 1b). This first HI-INPS experiment demonstrates the ability to measure local (dye) adsorption/desorption events, in the present case at the inner interface of the porous $\mathrm{TiO}_{2}$ layer, by monitoring time-resolved spectral shifts of the LSPR peak of the embedded plasmonic Au sensors. As complementing readout, we also monitored simultaneously the integral standard optical absorption signal from the dye, representing the total amount of adsorbed dye molecules. Thus both the total amount of adsorbed dye and the amount at the hidden inner interface were determined simultaneously as a function of time. A time constant of thousands of seconds was found for the dye impregnation front to reach the hidden interface. The experimental HI-INPS approach presented here thus offers a powerful tool to follow and optimize dye impregnation and, consequently, a tool to improve device performance in DSC systems. Furthermore it constitutes a general blueprint for experimental studies of diffusion processes in solid and nano/mesoporous materials.

INPS $^{5,6}$ employs gold plasmonic sensor nanoparticles (typically nanodisks) with dimensions in the range 50-500 $\mathrm{nm}$ for the diameter and at a constant $20 \mathrm{~nm}$ height on a transparent support. The sensors are coated with a thin (up to a few tens of $\mathrm{nm}$ ) dielectric spacer layer to tailor the surface chemistry of the INPS "sensor chip" and to protect the Au nanosensors from directly reacting with their environment (Figure 1a) and from structural reshaping. INPS sensors monitor changes occurring on the surface of the spacer layer or in its nanoenvironment via the locally strongly enhanced electromagnetic (EM) field, extending a few tens of nanometers from the spacer layer surface. Since, in the systems studied here, adsorbed dye molecules change the refractive index within the enhanced EM field region (the sensing volume), a spectral shift of the plasmon extinction peak is induced. This shift is used as the readout, in analogy with plasmonic biosensing. ${ }^{7}$ Specifically, we applied a peak-fitting procedure suggested by Dahlin et al., ${ }^{8}$ which routinely allows monitoring spectral shifts on the order of $1 \mathrm{~nm}$ or less with a reported resolution limit of $0.01 \mathrm{~nm}$. To determine the spectral peak position, a 20th degree polynomial fit is applied to the raw data around the LSPR peak, to then find the wavelength where the first derivative of the fitted polynomial is equal to zero. While the primary purpose of the polynomial fit is to reduce noise and provide a continuous analytical expression for the data, it also significantly simplifies data storage when large numbers of spectra are acquired during a measurement.

Figure la is a schematic illustration of the dense $\mathrm{TiO}_{2}$ INPS sensor chip structure used in the first part of the present work. It consists of $\mathrm{Au}$ nanodisks fabricated by hole-mask colloidal lithography (HCL), ${ }^{9}$ covered with a thin $(12-70 \mathrm{~nm})$ sputtered $\mathrm{TiO}_{2}$ film. A scanning electron microscopy (SEM) image of such a sample is shown in Figure 1c. Figure $1 \mathrm{~b}$ is the schematic illustration of the mesoporous thick $\mathrm{TiO}_{2}$ HI-INPS sensor chip structure used in the second part. It consists basically of the "standard" INPS $\mathrm{TiO}_{2}$ sensor from Figure la with a $12 \mathrm{~nm}$ thick dense and flat $\mathrm{TiO}_{2}$ spacer layer on the $\mathrm{Au}$ nanodisks, to provide suitable surface chemistry for attaching the thick mesoporous $\mathrm{TiO}_{2}$ film ontop (see Methods Section for details). An SEM image of a mesoporous $\mathrm{TiO}_{2}$ film is shown in Figure 1d. For the impregnation experiments, these samples were mounted in a flow cell and exposed to a Z907 dye solution (acetonitrile:tert-butanol, 1:1 volume ratio) under continuous white-light illumination, while the spectrally resolved extinction of the light was continuously monitored in a transmission configuration. A schematic drawing of the 
used setup is shown in Figure 1e (see Methods Section for details).

Figure 2a shows optical absorbance spectra of a sample comprising a $12 \mathrm{~nm}$ thick $\mathrm{TiO}_{2}$ film on the $\mathrm{Au}$ nanodisks measured, respectively, in air (blue curve), in pure solvent (green), and in the dye solution (dashed, red) after completion of the dye adsorption. The absorbance spectrum of the Z907 dye solution is also shown. The latter (blue, dashed-dotted), with a peak at ca. $525 \mathrm{~nm}$, is used to quantify dye injection/
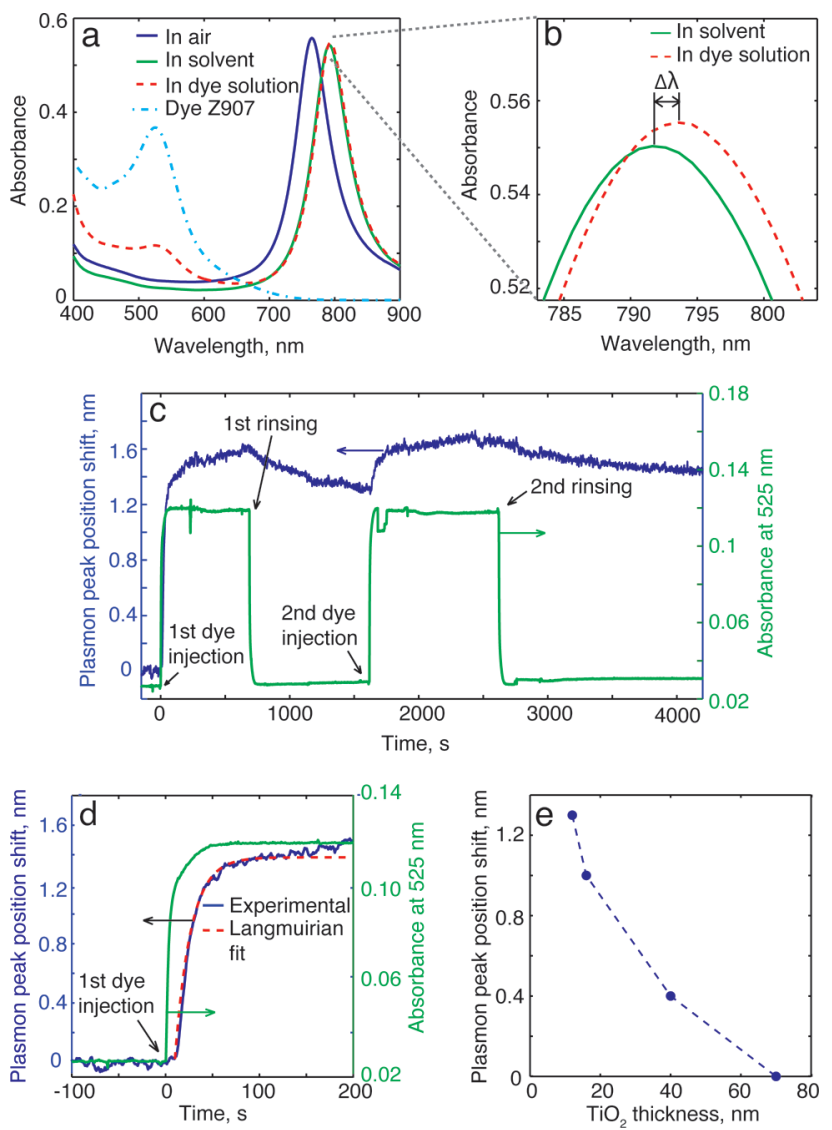

Figure 2. (a) Absorbance spectra of an INPS sample with a $12 \mathrm{~nm}$ $\mathrm{TiO}_{2}$ film for three cases: in air (blue curve), in the solvent (green), and in the dye solution (red, dashed). The absorbance spectrum of a solution of the Z907 dye is also displayed (blue, dashed-dotted). The latter was measured at a higher dye concentration than the one used for the INPS measurements, in order to better display the spectral features. Note the peak at $525 \mathrm{~nm}$ stemming from maximal light absorption by the dye at this particular wavelengt. This peak absorbance value at $525 \mathrm{~nm}$ was used to follow injection and removal of dye molecules into/from the bulk liquid. (b) Zoomed in spectra of the plasmon resonance peak position in the solvent and in dye solution. The spectral shift, denoted $\Delta \lambda$, is the quantity used to follow dye adsorption/desorption events on the $\mathrm{TiO}_{2}$ samples. (c) Temporal change of the plasmon peak position shift of the same sample (blue curve) and absorbance at the wavelength of $525 \mathrm{~nm}$ (in green) in a dye adsorption kinetics measurement. (d) Kinetics of the dye adsorption measured by INPS (the plasmon peak position shift) and the dye absorbance at $525 \mathrm{~nm}$ for the first dye injection step of panel c. A firstorder (irreversible) Langmuir adsorption kinetics fit to the plasmon peak position shift is also shown (red, dashed curve). (e) Plasmon peak position shift after $10 \mathrm{~min}$ of dye adsorption followed by $15 \mathrm{~min}$ of solvent rinsing, as a function of the $\mathrm{TiO}_{2}$ film thickness, mapping the extension of the sensing volume around the $\mathrm{Au}$ nanodisk sensor surface. rinsing using the Beer-Lambert law. ${ }^{10}$ The plasmon resonance position of the Au nanodisks shifts to the red (compared to air) when the sample is exposed to the solvent (Figure 2a), in agreement with the higher refractive index of the latter, ${ }^{7}$ and further red-shifts in the dye solution (Figure $2 b$ ). The latter shift is attributed to dye adsorption on the $\mathrm{TiO}_{2}$ surface.

Figure $2 \mathrm{c}$ shows the temporal evolution of the plasmon peak position (blue curve) during dye exposure up to near monolayer saturation. It also shows the subsequent rinsing with pure solvent. The green curve is the dye absorbance, deriving from the bulk liquid phase, and its rise/decline corresponds to dye injection/rinsing. The nonzero contribution seen at $t=0 \mathrm{~s}$ is caused by combined contributions to the absorbance from (i) an "extinction tail" related to the $\mathrm{TiO}_{2}$ and (ii) the $\mathrm{Au}$ nanoparticles. The whole experiment consisted of two sequential dye exposures, each followed by a rinsing step in pure solvent. Upon the first injection of the dye at $0 \mathrm{~s}$ the plasmon peak red-shifts rapidly, essentially following the rise of the dye signal originating from solution absorption (green) (but see Figure $2 \mathrm{~d}$ for minor details). Thereafter an additional slow increase of the signal was observed, to a total shift of ca. $1.6 \mathrm{~nm}$ (at ca. $700 \mathrm{~s}$ ). Rinsing at this time point resulted in immediate disappearance of the dye absorbance signal, as expected, and a slow spectral blue-shift of the INPS signal of ca. $0.3 \mathrm{~nm}$ after a total rinsing time of $900 \mathrm{~s}$. For this first part of the experiment, we attribute the initial red-shift of the plasmon peak position by $1.6 \mathrm{~nm}$ to adsorption of dye molecules onto the $\mathrm{TiO}_{2}$ film and the subsequent smaller blue-shift upon rinsing to desorption of a minor fraction of dye molecules that were reversibly bound, e.g., in aggregated form or at more weakly bound sites than the majority of the dye molecules.

In the later phase of the experiment, upon a second dye exposure at ca. $1600 \mathrm{~s}$ in Figure 2c, the peak red-shifted quite rapidly back to a value close to the one before the rinsing and then increased very slowly. A second rinsing (at ca. $2600 \mathrm{~s}$ ) resulted again in a slow spectral blue-shift (slower than upon the first rinsing), the final value ending at a slightly longer wavelength than after the first rinsing. This observation indicates that subsequent adsorption/rinsing steps generate a slight increase in the net amount of adsorbed dye, possibly due to rearrangements in the dye monolayer during rinsing, as has been observed for the dye N719. ${ }^{1}$ The initial fast adsorption at ca. $1600 \mathrm{~s}$ after the preceding slow desorption during rinsing supports this interpretation. In other words, if new irreversible binding sites are created, due to reorganization in the monolyer during the rinsing period, they are likely to be filled as rapidly as the initial binding sites on the unexposed surface, when a renewed exposure is made, i.e., with a signal rise time similar to that seen upon the first exposure.

Figure $2 \mathrm{~d}$ shows the very initial dye adsorption phase on a more expanded time scale and also the dye signal from the bulk solution. At this time resolution, the onset of the plasmon peak position shift occurs ca. $10 \mathrm{~s}$ after the onset of increasing absorbance at $525 \mathrm{~nm}$ (the maximum dye absorption wavelength), where the latter signals the presence of dye solution in the flow cell. The absorbance at $525 \mathrm{~nm}$ reaches its plateau value within ca. $50 \mathrm{~s}$ (when the dye concentration in solution is homogeneous in the flow cell) and then stays constant, whereas the plasmon shift continues to increase, indicating continued dye adsorption on the surface. The shape of the 'plasmon shift' curve after its onset fits first-order, essentially irreversible Langmuir adsorption, kinetics very well (Figure $2 \mathrm{~d}$ ). For longer times there is, as we saw above (Figure 
2c), also a small amount of reversibly bound molecules. The delayed onset of the INPS signal, compared to the bulk concentration signal, is most likely a transport effect. In other words, the bulk solution reaches its final concentration faster than it does in the boundary layer at the geometric INPS measurement point at the $\mathrm{TiO}_{2}$ surface. There may also be initial gradients in the boundary layer due to the fast adsorption influencing the dye adsorption rate (the blue curve) but not the total dye absorbance signal.

Since the main intention of this experiment with a dense $\mathrm{TiO}_{2}$ film was to test the sensitivity of the INPS method for dye adsorption and to obtain the time constant for monolayer formation for a flat surface, we refrain from a more detailed quantitative analysis of the dye adsorption kinetics until experiments with different flow rates etc. are made (as part of a forthcoming paper). The latter will be necessary to discriminate between transport effects and surface kinetics. It will also be useful for further scrutiny of desorption kinetics and rearrangement effects in the monolayer. We note, however, that the shape of the blue curve in Figure 1d suggests that formation of a saturated (mono)layer of dye molecules takes considerable time (of the order of $100 \mathrm{~s}$ at the used concentration) even on a flat $\mathrm{TiO}_{2}$ surface and that it can be altered and possibly improved by a series of adsorption/rinsing steps, as discussed above. The obtained results suffice to show that INPS is a very attractive method for such measurements.

In the next experiment we explored the INPS sensitivity for dye monolayer formation on $\mathrm{TiO}_{2}$ films with different thickness, as a preparation for the HI-INPS experiments on thick mesoporous $\mathrm{TiO}_{2}$ films reported below. This is motivated by the fact that the adsorbed dye molecules will be at a larger distance from the $\mathrm{Au}$ sensor particles in the HI-INPS experiments, and we therefore need an estimate of the sensing volume for this particular system. Figure 2e shows the plasmon peak shift after $10 \mathrm{~min}$ of dye adsorption, followed by $15 \mathrm{~min}$ of rinsing with solvent, for four samples with identical sensing nanoparticles but different dense $\mathrm{TiO}_{2}$ spacer layer thicknesses. The time scale was chosen to ascertain complete dye monolayer formation (saturation). The plasmon peak shift monotonously decreased with increasing dense $\mathrm{TiO}_{2}$ film thickness and went to zero at a thickness of around $70 \mathrm{~nm}$, yielding a good estimate of the penetration depth of the plasmonic sensing EM field into dense $\mathrm{TiO}_{2}$ and thus also into the 3D mesoporous $\mathrm{TiO}_{2}$ film studied below. The observed sensitivity decay is as expected, since the plasmon enhanced near-field around the nanoparticle in the dipolar approximation decays with distance $r$ from the nanoparticle surface as $1 / r^{3}{ }^{11}$ We note, however, that the decay length is expected to be slightly longer for the mesoporous $\mathrm{TiO}_{2}$ film, due to the presence of solvent (with lower index of refraction than $\mathrm{TiO}_{2}$ ) in the pores.

The sensing depth of ca. $70 \mathrm{~nm}$ implies that dye molecules from the solution above the $\mathrm{TiO}_{2}$ film (in addition to those adsorbed on the surface), in principle, also can be sensed. However, taking the area that one adsorbed dye molecule occupies on a $\mathrm{TiO}_{2}$ surface as $2 \mathrm{~nm}^{2}$ (a value of $1.8 \mathrm{~nm}^{2}$ was suggested for a similar dye $\mathrm{N} 719$ on rutile $\mathrm{TiO}_{2}{ }^{12}$ and the $\mathrm{Z} 907$ dye used here is slightly bulkier), we estimate that for the given dye solution concentration, the dye molecules from the solution within the sensing volume constitute only ca. $0.4 \%$ of those adsorbed on the flat $\mathrm{TiO}_{2}$ surface. This fraction is even smaller in the case of porous films, and thus we assume that the plasmon shift is almost entirely due to the adsorbed dye molecules.

We now turn to the second stage of our experiments and demonstrate the possibility to locally monitor the dye penetration and adsorption process inside a $10 \mu \mathrm{m}$ thick mesoporous $\mathrm{TiO}_{2}$ film by using HI-INPS (see Figures 1b,d). The mesoporous film was fabricated on a "standard" INPS sensor chip covered with a $12 \mathrm{~nm}$ compact $\mathrm{TiO}_{2}$ film acting as an adhesion layer (see Figure 1d and Methods Section for details). From the measurement of the sensing volume above (Figure 2e), we infer that this structure allows detection of processes taking place within at least ca. $50 \mathrm{~nm}$ above the 12 nm compact $\mathrm{TiO}_{2}$ adhesion layer, i.e., into the porous $\mathrm{TiO}_{2}$ structure deposited on top. Any dye adsorption well beyond this distance will pass unnoticed by the HI-INPS sensing, i.e., dye adsorption will only be detected in ca. $0.5 \%$ volume fraction of the porous film.

Figure 3a shows the HI-INPS response of a sensor featuring a $10 \mu \mathrm{m}$ thick mesoporous $\mathrm{TiO}_{2}$ layer on top. The plasmon
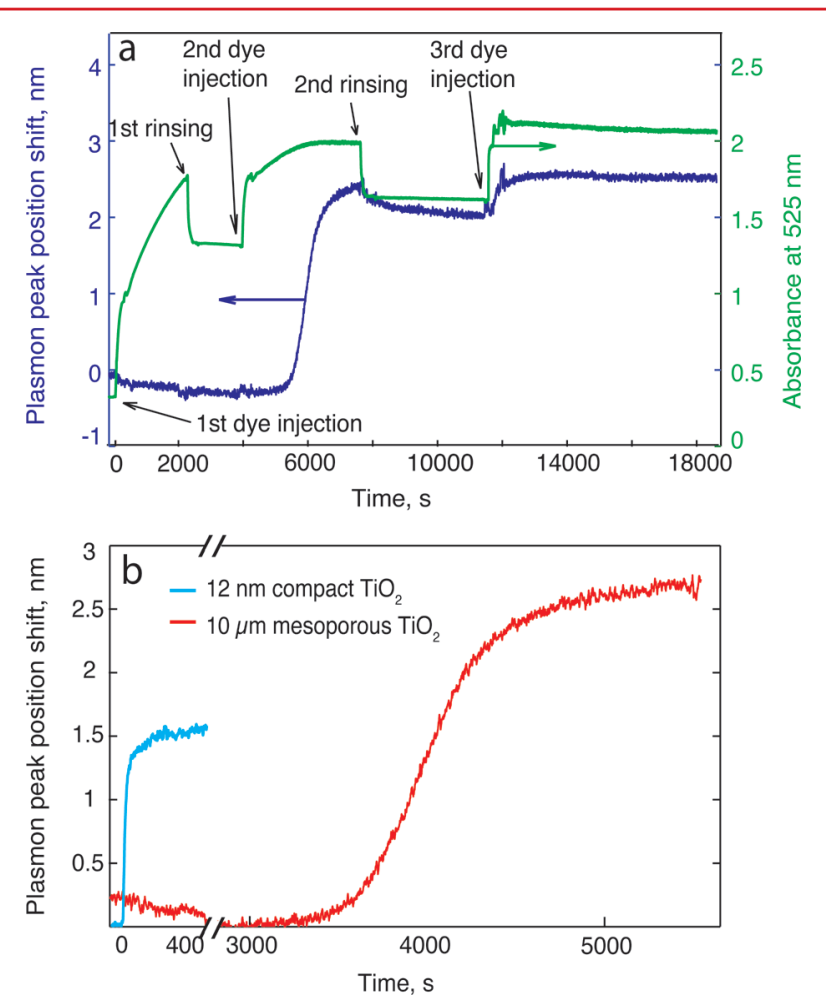

Figure 3. (a) Plasmon peak position shift and absorbance at $525 \mathrm{~nm}$ during three dye adsorption steps and two intermittent solvent rinsing steps for a $10 \mu \mathrm{m}$ thick mesoporous $\mathrm{TiO}_{2}$ film. (b) Comparison of time-resolved plasmon peak position shift signal (dye uptake kinetics) for the $12 \mathrm{~nm}$ dense flat $\mathrm{TiO}_{2}$ film (INPS) and for the $10 \mu \mathrm{m}$ mesoporous $\mathrm{TiO}_{2}$ film (HI-INPS), omitting the first solvent rinsing step in the latter case.

peak position (blue curve) is shown as a function of time during a dye exposure and with two intermediate rinsing steps. The corresponding absorbance at $525 \mathrm{~nm}$ (green curve) is also shown. Note that the dye absorbance signal in this case is composed of the sum of absorbance of dye molecules in the bulk solution above the $\mathrm{TiO}_{2}$ and (after some time) of the additional absorbance stemming from the adsorbed dye molecules in the mesoporous $\mathrm{TiO}_{2}$. Immediately upon the first injection of the dye solution, the absorbance at $525 \mathrm{~nm}$ 
rises rapidly as the dye enters the flow cell. A break point in the slope appeared at ca. $200 \mathrm{~s}$, which is when the flow cell had been filled completely with dye solution. Subsequently, the dye uptake by the porous $\mathrm{TiO}_{2}$ film caused a successively larger contribution to the absorbance signal, compared to the contribution from dye molecues in the bulk liquid phase. Note that, in contrast to the behavior of the flat $\mathrm{TiO}_{2}$ film, the plasmon peak position does not change during a long period after dye injection.

Rinsing the flow cell with solvent (after ca. $2300 \mathrm{~s}$ ) induced a clear decrease of the $525 \mathrm{~nm}$ absorbance as the dye solution was flushed out of the flow cell. Note, however, that the signal now stabilized at a significantly higher level than at the start of the experiment due to a substantial amount of dye being retained within the mesoporous film. The plasmon peak position has, however, not changed and maintains the same value as in the beginning of the experiment, both during the rinsing step and in the beginning of the subsequent second injection of dye solution at around $4000 \mathrm{~s}$. This indicates that no dye molecules have yet reached the hidden internal interface between the dense $\mathrm{TiO}_{2}$ adhesion layer on the $\mathrm{Au}$ nanodisk sensors and the mesoporous $\mathrm{TiO}_{2}$ film.

During the second exposure of the sample to the dye solution, the absorbance at $525 \mathrm{~nm}$ increased further and eventually approached a saturation value, which indicates that the mesoporous $\mathrm{TiO}_{2}$ layer has come close to being completely impregnated and saturated with dye molecules. Only now is there a very pronounced $2.7 \mathrm{~nm}$ red-shift of the plasmon peak, ca. $5000 \mathrm{~s}$ after the first dye injection step, with a total exposure time of $3400 \mathrm{~s}$ (rinsing time subtracted). This strikingly long delay between first dye injection and the appearance of a plasmon peak shift reflects the time period it takes for dye molecules to percolate from the top to the bottom of the mesoporous film. Only then are they locally detected by the plasmonic nanosensors. The red-shift of the plasmon peak position observed after ca. $60 \mathrm{~min}$ total dye exposure time can, therefore, be attributed to the "arrival" of the dye molecules at the internal, hidden $\mathrm{TiO}_{2}$-substrate interface.

The second rinse produced a small blue-shift similar to that found for the dense films. It is most likely also of the same origin, namely desorption of dye aggregates or desorption from a minoroity of weakly binding adsorption sites. This desorption signal corresponds to ca. $15 \%$ of the total HI-INPS signal monitored during uptake. We also note that the absorbance at $525 \mathrm{~nm}$ during the second rinsing was higher than during the first one, showing that additional dye molecules had been incorporated in the mesoporous film. Upon the third dye injection, the plasmon peak position again quickly red-shifted by a small amount (ca. 19\% of the total uptake signal) in a similar manner as was seen for the dense $\mathrm{TiO}_{2}$ film (fast initial uptake kinetics after rinsing/desorption) and ultimately stabilized (at ca. $2.5 \mathrm{~nm}$ ). The saturation value was higher than at the end of the second rinse, indicating that the dye monolayer film now is more densely packed than after the first and second dye exposures.

We have also performed a HI-INPS measurement with a lower dye concentration ( 0.1 vs $0.3 \mathrm{mM}$ ) but otherwise identical conditions as the measurement discussed above (on the same sample, which was cleaned from dye molecules, see Methods Section and Supporting Information for details). For the $0.1 \mathrm{mM}$ solution, it took ca. $6400 \mathrm{~s}$ for dye molecules to reach the bottom of the $\mathrm{TiO}_{2}$ film. This is about twice as long as for the case of the $0.3 \mathrm{mM}$ solution and very reasonable when an impregnation via a diffusion front is considered. A detailed study of the dye diffusion in the mesoporous $\mathrm{TiO}_{2}$ matrix, including analysis of the dependence of the impregnation time on dye concentration and other parameters, is the subject of a forthcoming publication.

In Figure $3 b$ we compare the dye adsorption kinetics on the $12 \mathrm{~nm}$ thick dense and flat $\mathrm{TiO}_{2}$ film and the $10 \mu \mathrm{m}$ thick mesoporous $\mathrm{TiO}_{2}$ sample, omitting the rinsing step. On the dense film the adsorption occurs almost instantaneously; the observed small delay most likely being bulk transport related, as discussed in connection with Figure 2 above. Clearly, it takes much longer time for the onset and saturation of the plasmon shift in the case of the porous film. The resulting plasmon peak shifts are ca. $1.6 \mathrm{~nm}$ in the dense and ca. $2.7 \mathrm{~nm}$ in the mesoporous film case. The long delay time in the latter case is due to the short sensing depth of the plasmonic sensing field (see Figure 2e), which only detects dye molecules as they arrive within that sensing depth, i.e., in the ca. $0.5 \%$ bottom part of the mesoporous $\mathrm{TiO}_{2}$ sample, close to the inner interface. From the estimated sensing depth of ca. $70 \mathrm{~nm}$ and the known $\mathrm{TiO}_{2}$ film porosity and surface area, we calculate that there are roughly 4-5 times more molecules in the plasmonic nanoparticle sensing volume for the case of the porous film compared to the dense, flat film system. However, most of the former molecules are further away from the plasmon nanoparticles than in the case of a dense $\mathrm{TiO}_{2}$ film and therefore contribute less to the plasmon peak position shift. The ratio of the observed values for the two cases, about twice larger signal for the mesoporous sample, is therefore very reasonable.

As a last step, we want to highlight the local sensitivity of the HI-INPS measurement in contrast to the integrating dye loading measurement techniques, such as the optical spectroscopy or QCM-D. For this purpose, the contribution to the absorbance at $525 \mathrm{~nm}$ signal, stemming from the dye molecules in the solution above the $\mathrm{TiO}_{2}$, was subtracted from the total signal (Figure 4a). It becomes very clear that the shift in the LSPR peak position basically starts when the absorbance at 525 $\mathrm{nm}$ signal is very close to saturation (the decrease in the absorbance after ca. $6000 \mathrm{~s}$ is likely caused by photobleaching and/or some desorption of dye due to the long rinsing of dye molecules). As a next step, we plot in Figure $4 \mathrm{~b}$ the plasmon peak position shift as a function of the dye absorbance (at 525 $\mathrm{nm}$ ) originating from the dye molecules in the $\mathrm{TiO}_{2}$ only, to eliminate time as an explicit parameter. In the resulting graph, the long horizontal part of the (blue) curve shows very convincingly that in this period, only the absorbance at $525 \mathrm{~nm}$ changes, due to successively deeper penetration of the dye adsorption into the mesoporous $\mathrm{TiO}_{2}$ structure. The HI-INPS signal is basically constant. In contrast, the steep, almost vertical rise of the curve illustrates that dye molecules have now reached the internal hidden interface and saturate also this part of the mesoporous film, which lies within the sensing volume of the HI-INPS sensor. The associated contribution to the total dye uptake, measured by the absorbance signal, is very small, and therefore, the steep rise is almost vertical.

In summary, we have demonstrated local, in situ, real time kinetics measurements of dye molecule adsorption for dense and mesoporous $\mathrm{TiO}_{2}$ films by monitoring shifts in the plasmon peak position of $\mathrm{Au}$ sensor nanoparticles integrated into the $\mathrm{TiO}_{2}$, according to the INPS and novel HI-INPS schemes, respectively. The kinetics and mechanistic details about the dye adsorption process that can be obtained in this 

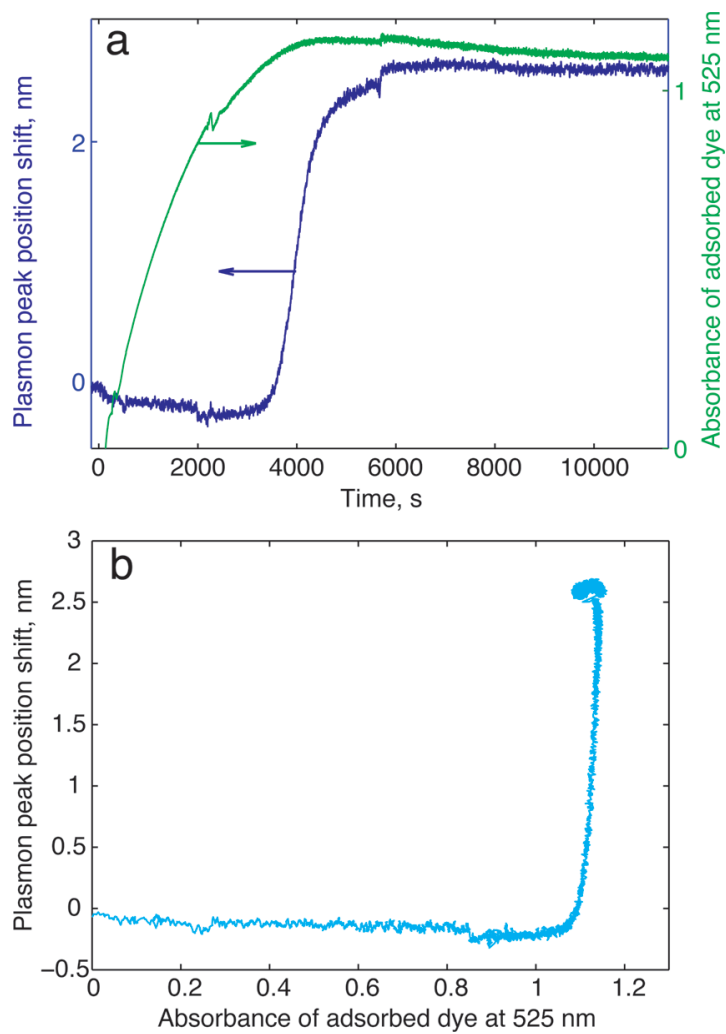

Figure 4. (a) Same data as presented in Figure 3a but with rinsing steps omitted. The peak shift data from the HI-INPS measurement are plotted together with the dye absorbance at $525 \mathrm{~nm}$ signal (with the contribution from the dye molecules in the solution above the $\mathrm{TiO}_{2}$ film subtracted). (b) Plasmon peak position shift plotted versus absorbance of the dye adsorbed in the mesoporous $\mathrm{TiO}_{2}$ film. The steep rise of the blue curve is signaling arrival of dye molecules at the inner interface of the mesoporous $\mathrm{TiO}_{2}$ film and thus within the sensing volume of HI-INPS.

way are unique and important for DSC research. They have the potential to significantly contribute to the development of DSCs with higher efficiencies.

For dense films, adsorption/desorption (rinsing) kinetics, saturation times and adsorbed dye amounts can be studied in detail with minimized influence of transport effects, e.g., as functions of surface properties and bulk liquid composition. Specifically, we observed an adsorption time scale to reach saturation of the order of $100 \mathrm{~s}$ for the used dye concentration. We further observed desorption upon rinsing and a net increase in the plasmon peak position shift (corresponding to increased uptake) after a second dye adsorption step, which may be attributed to the formation of a more compact dye monolayer, in agreement with the recent findings of Bazzan et al. ${ }^{1}$

Using the novel HI-INPS methodology, we performed for the fist time measurements of the dye adsorption kinetics locally inside thick (10 $\mu \mathrm{m}$ in the present case) mesoporous $\mathrm{TiO}_{2}$ films. This was made possible by placing plasmonic $\mathrm{Au}$ sensor nanoparticles at the hidden inner interface between a mesoporous $\mathrm{TiO}_{2}$ layer, as used as photoanode in a DSC, and the support (corresponding to the electron collecting electrode in a DSC). For this system with an average pore size of $20 \mathrm{~nm}$, a total dye impregnation time of ca. 60 min was identified for the used dye concentration via the time delay between dye injection and the response of the plasmonic sensors at the support- $\mathrm{TiO}_{2}$ interface. Simultaneous measurements of the
INPS signal and the dye absorbance signal at $525 \mathrm{~nm}$ made it possible to obtain time-resolved and independent information about the interfacial and the total amount of dye adsorbed in the mesoporous $\mathrm{TiO}_{2}$ film (the latter in analogy to widely used optical absorption spectroscopy) and dye concentration in the bulk solution. Indications for a higher dye molecule density on both the dense and the mesoporous $\mathrm{TiO}_{2}$ surfaces after intermittent rinsing and readsorption steps were found.

In a broader perspective diffusion or combined diffusion/ adsorption/desorption kinetics is relevant for many other applications, where molecules diffuse through porous or solid materials to a hidden, internal interface. Owing to its generic nature, the INPS and HI-INPS techniques presented here complement existing methodologies to study diffusion in porous materials ${ }^{13}$ and have great potential to provide new insights into diffusion kinetics in, e.g., porous catalyst systems, gas adsorbing/absorbing materials, metal-organic frameworks, and slow release systems. ${ }^{14-16}$

Methods. Gold $(\mathrm{Au})$ plasmonic nanodisks with a diameter of $110 \mathrm{~nm}$ and a height of $20 \mathrm{~nm}$ were fabricated on circular soda lime glass slides by HCL. ${ }^{9}$ The compact flat $\mathrm{TiO}_{2}$ films were deposited on top of the $\mathrm{Au}$ nanodisks by reactive sputtering from a Ti target (FHR MS150 sputter system). For the mesoporous $\mathrm{TiO}_{2}$ film, a soda lime glass substrate with $\mathrm{Au}$ nanodisks was first coated with a $12 \mathrm{~nm} \mathrm{TiO}_{2}$ film by sputtering. Subsequently, a mesoporous film of $\mathrm{TiO}_{2}$ was prepared by screen printing ${ }^{17}$ a colloidal $\mathrm{TiO}_{2}$ paste onto the previously modified substrates and subsequent sintering at 500 ${ }^{\circ} \mathrm{C}$ for $1 \mathrm{~h}$ in air, resulting in the final thickness of ca. $10 \mu \mathrm{m}$. The film had the average particle size of $20 \mathrm{~nm}$, an average pore size of $23 \mathrm{~nm}$, and a porosity of $69 \%$, which was determined from BET nitrogen desorption measurements. A dye removal procedure, applied in order to reuse samples, consisted of a 30 min long desorption in a $0.1 \mathrm{M}$ solution of TBAOH in DMF, followed by a $30 \mathrm{~min}$ rinsing in deionized water and subsequent sintering at $400{ }^{\circ} \mathrm{C}$ for $30 \mathrm{~min}$ in air.

A $0.05 \mathrm{mM}$ solution of the $\mathrm{Ru}$ dye $\mathrm{Z} 907$ in acetonitrile:tertbutanol (1:1 volume ratio) was used for the measurements on the compact $\mathrm{TiO}_{2}$ films, and a concentration of $0.3 \mathrm{mM}$ was used for the porous film experiment. The solutions were pumped through the flow cell (Figure 1e) using a peristaltic pump at a flow rate of $0.3 \mathrm{~mL} / \mathrm{min}$. The internal volume of the flow cell is approximately $200 \mu \mathrm{L}$. The extinction spectra were recorded using a white lamp and a CCD detector from Avantes at a rate of 1 spectrum per second $(1 \mathrm{~Hz})$ and analyzed using the Insplorer software (Insplorion AB, Göteborg, Sweden), exploiting the analysis routines suggested by Dahlin et al. ${ }^{8}$

\section{ASSOCIATED CONTENT}

\section{S Supporting Information}

An HI-INPS measurement of the $10 \mu \mathrm{m}$ thick mesoporous $\mathrm{TiO}_{2}$ film using a lower dye concentration of $0.1 \mathrm{mM}$, and raw absorbance spectra in solvent and during the dye adsorption for the $12 \mathrm{~nm}$ dense $\mathrm{TiO}_{2}$ film and the $10 \mu \mathrm{m}$ thick mesoporous $\mathrm{TiO}_{2}$ film samples. This material is available free of charge via the Internet at http://pubs.acs.org.

\section{AUTHOR INFORMATION}

\section{Corresponding Author}

*clangham@chalmers.se; kasemo@chalmers.se

Notes

The authors declare no competing financial interest. 


\section{ACKNOWLEDGMENTS}

We acknowledge financial support from the Swedish Research Council project 2010-4041 "Nanoplasmonics for Nanomaterials Science” (C.L.), the Swedish Energy Agency project dnr. 0189-1 "Nanoscience and Nanotechnology for Sustainable Energy and Environment" (V.G. and B.K.), and the Swiss National Science Foundation grant no. 200020_125163 (L.P.H.). We also thank Pascal Comte for paste preparation, Shaik Mohammed Zakeeruddin for Z907 dye, and Hauke Harms and Nicolas Tétreault for fruitful discussions.

\section{REFERENCES}

(1) Bazzan, G.; Deneault, J. R.; Kang, T.-S.; Taylor, B. E.; Durstock, M. F. Adv. Funct. Mater. 2011, 21, 3268-3274.

(2) Fattori, A.; Peter, L. M.; McCall, K. L.; Robertson, N.; Marken, F. J. Solid State Electrochem. 2010, 14, 1929-1936.

(3) Harms, H. A.; Tetreault, N.; Gusak, V.; Kasemo, B.; Grätzel, M. 2012, submitted.

(4) Peic, A.; Staff, D.; Risbridger, T.; Menges, B.; Peter, L. M.; Walker, A. B.; Cameron, P. J. J. Phys. Chem. C 2011, 115, 613-619.

(5) Langhammer, C.; Larsson, E. M.; Kasemo, B.; Zoric, I. Nano Lett. 2010, 10, 3529-3538.

(6) Langhammer, C.; Zhdanov, V. P.; Zoric, I.; Kasemo, B. Phys. Rev. Lett. 2010, 104, 135502.

(7) Anker, J. N.; Hall, W. P.; Lyandres, O.; Shah, N. C.; Zhao, J.; Van Duyne, R. P. Nat. Mater. 2008, 7, 442-453.

(8) Dahlin, A. B.; Tegenfeldt, J. O.; Höök, F. Anal. Chem. 2006, 78, 4416-4423.

(9) Fredriksson, H.; Alaverdyan, Y.; Dmitriev, A.; Langhammer, C.; S., S. D.; Zäch, M.; Kasemo, B. Adv. Mater. 2007, 19, 4297.

(10) Katoh, R.; Yaguchi, K.; Murai, M.; Watanabe, S.; Furube, A. Chem. Phys. Lett. 2010, 497, 48-51.

(11) Jain, P. K.; El-Sayed, M. A. Chem. Phys. Lett. 2010, 487, 153164.

(12) Kavan, L.; Grätzel, M.; Gilbert, S. E.; Klemenz, C.; Scheel, H. J. J. Am. Chem. Soc. 1996, 118, 6716-6723.

(13) Chmelik, C.; Kärger, J. Chem. Soc. Rev. 2010, 39, 4864-4884.

(14) Li, J.-R.; Kuppler, R. J.; Zhou, H.-C. Chem. Soc. Rev. 2009, 38, $1477-1504$.

(15) Melde, B. J.; Johnson, B. J. Anal. Bioanal. Chem. 2010, 398, $1565-1573$

(16) Shekhah, O.; Liu, J.; Fischer, R. A.; Wöll, C. Chem. Soc. Rev. 2011, 40, 1081-1106.

(17) Ito, S.; Murakami, T. N.; Comte, P.; Liska, P.; Graetzel, C.; Nazeeruddin, M.; Graetzel, M. Thin Solid Films 2008, 516, 46134619. 